\title{
Firing Activities in Fractional-Order Hindmarsh-Rose Neuron with Multistable Memristor as Autapse
}

\author{
Zhijun Li ( $\square$ lizhijun_320@163.com ) \\ Xiangtan University https://orcid.org/0000-0003-0311-4532 \\ WenQiang Xie \\ Xiangtan University \\ Jinfang Zeng \\ Xiangtan University \\ Yicheng Zeng \\ Xiangtan University
}

\section{Research Article}

Keywords: Fractional-order, Multistable, Neuron, Firing, Local active memristor

Posted Date: November 18th, 2021

DOI: https://doi.org/10.21203/rs.3.rs-1077040/v1

License: (c) (i) This work is licensed under a Creative Commons Attribution 4.0 International License.

Read Full License

Version of Record: A version of this preprint was published at Chinese Physics B on April 11th, 2022. See the published version at https://doi.org/10.1088/1674-1056/ac65f7. 


\title{
Firing activities in fractional-order Hindmarsh-Rose neuron with multistable memristor as autapse
}

\author{
Zhijun $\mathrm{Li}^{*}{ }^{1}$, Wenqiang Xie ${ }^{1}$, Jinfang Zeng ${ }^{2}$, Yicheng Zeng ${ }^{2}$ \\ 1. School of Automation and Electronic Information, Xiangtan University, Xiangtan, Hunan 41105, China
}

2. School of Physics and Optoelectronics, Xiangtan University, Xiangtan, Hunan 41105, China

\begin{abstract}
Compared with integer order neurons, fractional-order neuron model can more accurately describe the firing behavior of biological neurons. Considering the fact that memristors have the characteristics similar to biological synapses, a fractional-order multistable memristor is firstly proposed in this study. It is verified that the fractional-order memristor has multiple local active regions and multiple stable hysteresis loops, and the influence of fractional order on its nonvolatility is also revealed. Then by considering the fractional-order memristor as an autapse of HR neuron model, a fractional-order memristive neuron model is developed. The effects of the initial value, external excitation current, coupling strength and fractional order on the firing behavior are discussed by time series, phase diagrams, Lyapunov exponents and inter spike interval (ISI) bifurcation diagrams. Three coexisting firing patterns, including irregulate A-periodic bursting, Aperiodic bursting and chaotic bursting, dependent on the memristor initial values are observed. It is also revealed that the fractional order can not only induce the transition of firing patterns, but also change the firing frequency of the neuron. Finally, a neuron circuit with variable fractional order is designed to verify the numerical simulations.
\end{abstract}

Keywords: Fractional-order; Multistable; Neuron; Firing; Local active memristor

\section{Introduction}

As an important basic unit of the nervous system, biological neuron has rich and complex firing activities. In order to study the firing patterns and operating mechanisms of biological

\footnotetext{
* Corresponding author.

E-mail address: lizhijun_320@163.com
} 
neurons, many mathematical models have been proposed to describe and simulate unit neurons. The classic paradigm is the Hodgkin Huxley (HH) model [1] proposed by British physiologist and biophysicist Hodgkin and Huxley in 1952. Subsequently, some simplified models, such as Hindmarsh-Rose (HR) [2], FitzHugh-Nagumo model (FHN) [3], Izhikevich [4], and MorrisLecar models (ML) [5] were derived from the classic HH model. Synapses are the key parts of functional connection and information transmission between neurons, so how to model the function of biological synapses has become an urgent problem. Memristor [6-7] devices are considered to be the optimal solution to simulate biological synapses due to their good biomimetic characteristics [8-9] such as low power consumption, non-volatile, plasticity and nano size. Thus, considering memristors as coupling synapses to construct nervous systems has become a hot topic in the field neurodynamics. For example, Chen et al. proposed a Hopfield neural network with non-ideal memristor as coupling synapse, in which hyper-chaotic phenomenon was observed [10]. Bao et al. proposed a multi-neuron network with unbalanced memristor coupling synapse, revealing that it has various collective behaviors such as incoherent, coherent, incomplete synchronization and chimeric states [11]. Refs [12-14] also studied the collective dynamic behavior of multiple neurons with memristors as coupling synapses.

As we all know, fractional calculus is an extension of integral calculus, which can accurately depict long memory and hereditary properties of dynamical process in object world. In recent years, fractional calculus has been widely used in physics, biomedicine, biophysics and other fields [15-17]. In neurodynamics, fractional differentiation can predict stimuli, improve information processing ability [18]. Therefore, fractional-order neuron models can mimic the electrical activity of biological neuron more reasonably. In Ref [19], fractional calculus was used to model complex biological systems in a direct and rigorous manner, accurately describing the propagation of subthreshold nerves. In Ref [18], it was found that the multi-time scale adaptation of single rat neocortical pyramidal neurons could be well simulated by fractional calculus. The study on dynamics of fractional-order neurons is also a hot spot in nonlinear realm. Yun et al. found hidden periodic and chaotic bursting in fractional-order HR neuron model by fast-slow analysis [20]. Alidousti et al. revealed the different firing patterns of fractional-order FNH neuron model and the related bifurcation mechanisms are also explored 
[21]. The dynamics and chaotic synchronization of two fractional coupled HR neurons with time delay under electromagnetic radiation were explored by Meng et al [22]. Their research results show that the fractional order has an important influence on the firing patterns. Similarly, the effects of fractional order on synchronization of neuronal networks under different external conditions were reported in [23-25].

Local activity is the origin of complexity [26]. In 2014, Chua proposed the first locally active memristor [27]. Compared with ideal memristors, local active memristors have more complex nonlinear dynamic characteristics [28-30]. The multi-stability characteristic of local active memristor [31] make it a good means to study the conversion and coexistence of various firing patterns in neuron models. Thus, local active memristor based neurons and neuronal networks have been extensively studied in recent years. For example, Li et al. constructed a bistable neural network based on local active memristor, and revealed the switching and bifurcation behaviors of firing patterns in the neural network [32]. Lin et al. constructed an HR neural network based on a tristable locally active memristor, and found that the proposed neural network produces three multi-stable phenomena with four different firing patterns simultaneously [33]. A model of HR neuron with four-stable locally active memristor as selfsynapses was proposed by Li et al. and they found that four stable firing patterns in the proposed neuron can be switched freely by selecting appropriate initial conditions [34]. With further development of memristor research, locally active memristor has been extended to fractionalorder form, and the related research has been carried out. Xie et al. proposed a fractional-order memristor with infinite locally active interval and coupled it to Chen's chaotic system. They found that the system presents different states under different fractional orders [35]. However, so far, there is no relevant research on fractional-order neuron model with locally active memristor.

Inspired by the above studies, a novel fractional multistable locally active memristor is proposed in this paper, and a new neuron model is constructed by coupling it with fractionalorder HR neuron. The main contributions of this work are summarized as follows. Firstly, a novel fractional-order locally active memristor is developed. Its nonvolatile memory and locally active characteristics, initial value-dependent coexisting pinched hysteresis loops and fractional order-dependent electrical behavior are analyzed. Secondly, firing activities of the 
fractional-order memristive neuron model under different external conditions are studied. Finally, physical implementation circuit of the fractional-order neurons is proposed and the circuit simulation verification is performed.

The layout of this paper is as follows. In Sec. 2, a new fractional tristable locally active memristor is introduced and its related characteristics are studied in detail. In Sec. 3, a 4D fractional-order neuron model, by considering the fractional-order multistable locally active as an autapse of 3D-HR neuron, is constructed, and the stability of the neuron model is analyzed. In Sec. 4, the influences of coupling strength, external excitation current and fractional order on the fractional-order neuron model are discussed. In Sec. 5, the circuit simulation of the proposed fractional-order neuron model is carried out, and the conclusion is given in the last section.

\section{Model and characteristics of fractional-order multistable memristor}

It is well known that there are three definitions for fractional calculus, i.e. GrünwaldLetnikov (GL) definition, Riemann-Liouville (RL) definition and Caputo definition. Among them, Caputo definition is very suitable for real world applications due to its initials with the same form as those of its integer order counterparts. In this paper, the Caputo definition, thus, is used to characterize the neuron model and the Caputo definition of $f(t)$ is described as:

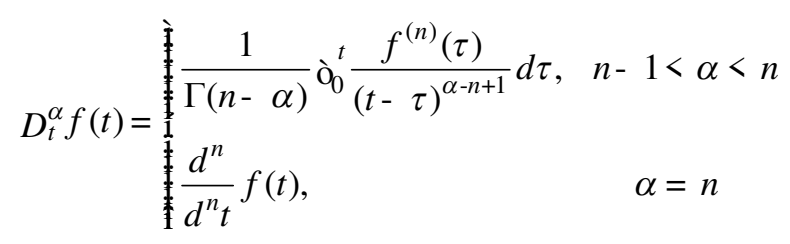

where, $0<\alpha<1$ is the fractional order, $n$ is the first integer which is not less than $\alpha$ and $\Gamma(\cdot)$ is Euler gamma function.

\subsection{Fractional-order multistable memristor model}

In this paper a new fractional-order multistable local active memristor is proposed which is defined by the state-dependent Ohm's law

$$
i=G(\varphi) v=n \varphi v
$$

where $i$ and $v$ represent the input current and input voltage, $\varphi$ the is internal state variable and $G(\varphi)=n \varphi$ is the defined memductance function. The corresponding state equation can be described as 


$$
D_{t}^{\alpha} \varphi=(\operatorname{sgn}(\varphi+2)+\operatorname{sgn}(\varphi-2)-0.5 \varphi)+\beta v
$$

where $\beta$ is the memristive parameter. It is worth noting that the fractional-order state equation is constructed with sign functions which are related to the internal state $\varphi$ and the terminal voltage $v$. Compared with the existing locally active memristors whose state equations are implemented with polynomial functions [28, 30-31], the proposed fractional-order locally active memristor is more convenient for circuit implementation with low cost. In the following, we will study the basic characteristics of the memristor from the aspects of pinched hysteresis loop, nonvolatile memory and locally active characteristics.
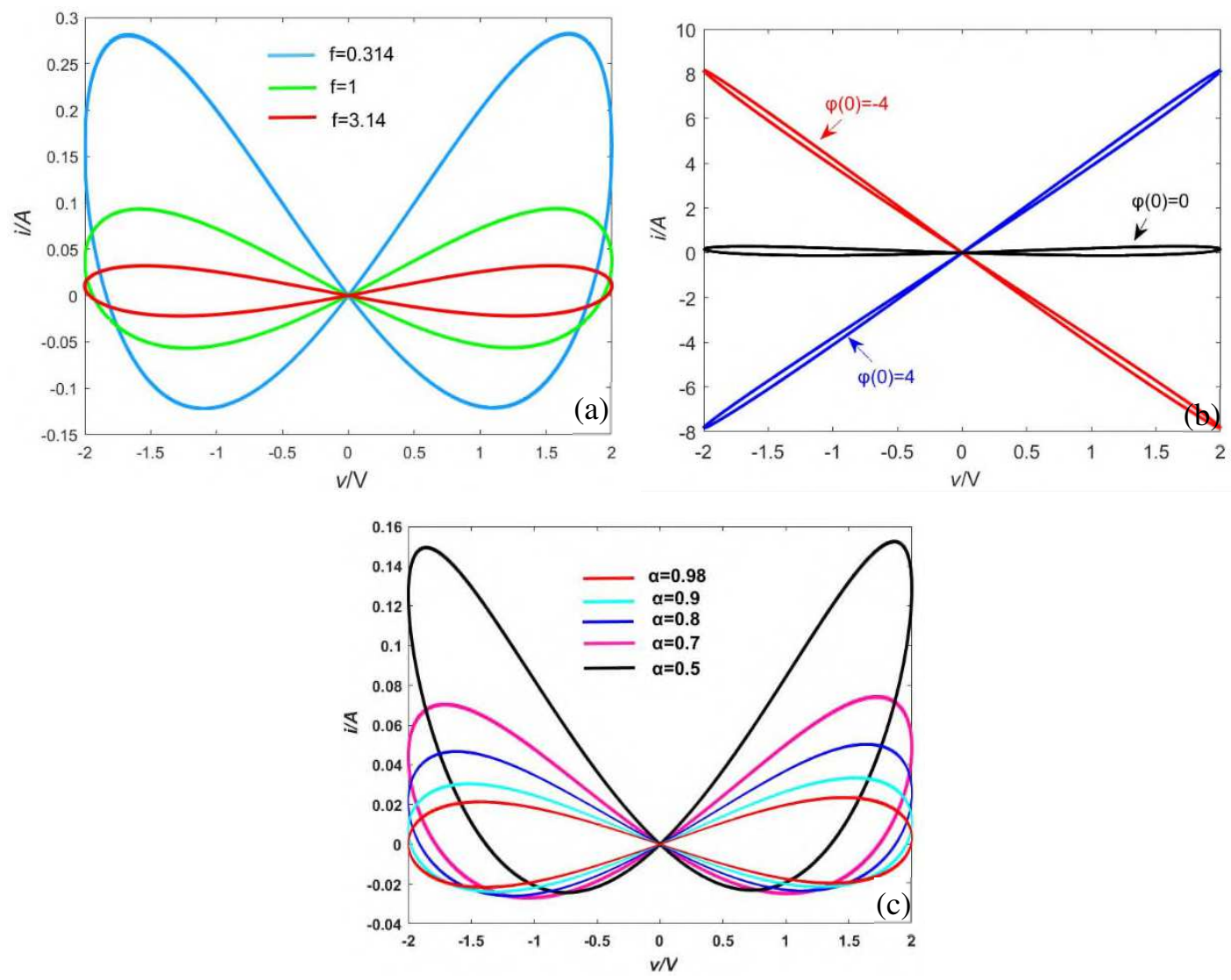

Fig.1 Pinched hysteresis loops (a) Hysteresis loops with respect to frequency, (b) pinched hysteresis loops with different initial values (c) pinched hysteresis loops with different fractional orders

\subsection{Pinched hysteresis loop}

Pinched hysteresis loop is one of the important characteristics of memristor. When the parameter $\beta=0.2$ and the order $\alpha=0.9$, a sinusoidal voltage $v=A \sin (2 \pi F t)$ with amplitude $A$ $=2$ is applied to the memristor, the pinched hysteresis loops with different excited frequencies are shown in Fig.1(a). It can be seen from Fig.1(a) that when the input frequency $F$ increases 
from 0.314 to 3.14 , the pinch hysteresis loop gradually decreases. Once the excited frequency approaches infinity, the hysteresis loop becomes a single valued function.

In addition, when $A=2$ and $F=0.314$ remain unchanged, three stable pinched hysteresis loops can be obtained under different initial states $\varphi_{0}=-4,0$, and 4, as shown in Fig.1(b). In order to study the influence of fractional order $\alpha$ on the pinched hysteresis loop, keep $A=2, F$ $=3.14$, and memristor initial value $\varphi_{0}=0$, the fractional order $\alpha$ is selected as $0.98,0.9,0.8,0.7$ and 0.5, respectively, the hysteresis loops of the memristor are shown in Fig.1(c). It is observed that the smaller the fractional order is, the larger the area of the hysteresis loop is, and thus the better memory of the memristor is [36].

\subsection{Nonvolatile memory and locally active characteristics}

Non-volatility is also a key characteristic of memristor, which means memristor can keep its latest memductance value when the power is switched off. Usually, the POP (Power-Off Plot) is used to judge whether an electronic device is nonvolatile [37]. Let the input voltage $v=0$, and the state equation (3) becomes:

$$
D_{t}^{\alpha} \varphi=\operatorname{sgn}(\varphi+2)+\operatorname{sgn}(\varphi-2)-0.5 \varphi
$$

When order $\alpha=0.9$, the POP of the fractional memristor is shown in Fig.2(a). It can be seen from Fig.2(a) that the POP has five intersections with the $x$-axis, among which three intersections $E_{1}(-4,0), E_{3}(0,0)$ and $E_{5}(4,0)$ are negative slope intersections and the other two intersections $E_{2}(-2,0)$ and $E_{4}(2,0)$ have an infinite positive slope. It should be noted that the five intersections are the equilibrium points of the memristor. The arrows near the intersection in Fig.2 (a) indicate the moving direction of the POP. It is easy to know that the three negative slope intersections are stable equilibrium points and the other two positive slope intersections are unstable equilibrium points. For arbitrary initial value $\varphi(0)$, the memristor will eventually tend to one of the three stable equilibrium points, Namely

$$
\left\{\begin{array}{lc}
\varphi=\varphi\left(\mathrm{E}_{1}\right)=-4, & \varphi(0)<-2 \\
\varphi=\varphi\left(\mathrm{E}_{3}\right)=0, & -2<\varphi(0)<2 \\
\varphi=\varphi\left(\mathrm{E}_{5}\right)=4, & 2<\varphi(0)
\end{array}\right.
$$

When $n=1$, the corresponding memductance values are 


$$
\left\{\begin{array}{lr}
w\left(\varphi\left(E_{1}\right)\right)=\varphi\left(Q_{1}\right)=-4, & \varphi(0)<-2 \\
w\left(\varphi\left(E_{3}\right)\right)=\varphi\left(Q_{3}\right)=0, & -2<\varphi(0)<2 \\
w\left(\varphi\left(E_{5}\right)\right)=\varphi\left(Q_{5}\right)=4, & 2<\varphi(0)
\end{array}\right.
$$

Thus, the proposed memristor is nonvolatile and it acts as a discrete memory device, which can encode three memory states.
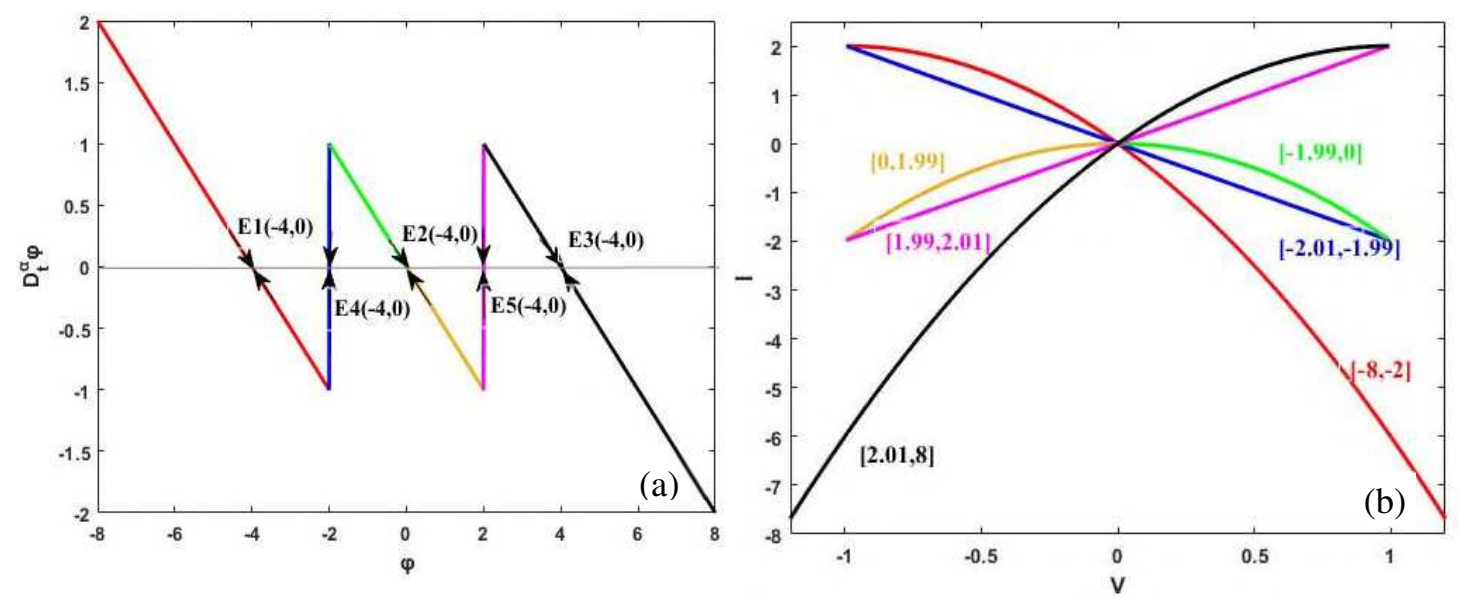

Fig.2 (a) The power-off-plot (POP) and (b) The DC V-I curve of the local active memristor

DC $V-I$ curve is an important tool to judge whether the memristor has local activity. When the DC $V$-I curve of the memristor has a negative slope, it is locally active [38]. Let $D_{\mathrm{t}}^{\alpha} \varphi=0$, Eqs. (2) and (3) can be written as

$$
\begin{gathered}
V=(\operatorname{sgn}(\phi-2)-\operatorname{sgn}(\phi+2)-0.5 \phi) \\
I=V \phi=\phi(\operatorname{sgn}(\phi-2)-\operatorname{sgn}(\phi+2)-0.5 \phi)
\end{gathered}
$$

where $V, I$ denote DC voltage and current, respectively, and $\Phi$ is a variable equilibrium state which satisfies $D_{t}^{\alpha} \varphi=(\varphi=\Phi)=0$. By virtue of Eqs. (6) and (7), the DC V-I curve of the memristor is obtained and shown in Fig.2 (b). Observation from Fig. 2(b) that the slopes of the red, green and blue curves are negative, so the fractional memristor is locally active and suitable to realize electronic synapses.

\section{3、 Fractional-order memristive neuron model and its stability analysis}

\subsection{Fractional-order memristive HR model}

According to the Caputo definition, the fractional-order HR neuron model can be described as the following differential equations 


$$
\left\{\begin{array}{l}
D_{t}^{\alpha} x=y-a x^{3}+b x^{2}-z+I \\
D_{t}^{\alpha} y=c-d x^{2}-y \\
D_{t}^{\alpha} z=r(s(x+1.6)-z)
\end{array}\right.
$$

where $x$ represents the membrane potential, $y$ is the recovery or spiking variable, $z$ is the adaptation variable, $I$ is an external excitation current, $\alpha$ is the system order and $a, b, c, d, r, s$ are system parameters. In this study, we consider the proposed locally-active memristor governed by (2) and (3) as an autapse of the fractional-order HR neuron (9), then a new 4D neuron model is developed, which is formulated as

$$
\left\{\begin{array}{l}
D_{t}^{\alpha} x=y-a x^{3}+b x^{2}-z+I+k \varphi x \\
D_{t}^{\alpha} y=c-d x^{2}-y \\
D_{t}^{\alpha} z=r(\mathrm{~s}(x+1.6)-z) \\
D_{t}^{\alpha} \varphi=(\operatorname{sgn}(\varphi+2)+\operatorname{sgn}(\varphi-2)-0.5 \varphi)+\beta x
\end{array}\right.
$$

where $k$ is coupling strength, I represent the external excitation current. In this paper, the parameters are determined as $a=1, b=3, c=1, d=5, r=0.0021, s=4$, and $\beta=0.2$. The effects of external excitation current $I$, fractional order $\alpha$ and coupling strength $k$ on the firing dynamics of the memristive neuron model are discussed in detail in the Section 4.

\subsection{Stability analysis of equilibrium points}

In order to analyze the stability of the equilibrium point of the fractional-order neuron, the following theorem is introduced.

Lemma 1[39]: For a fractional-order system with order $0<\alpha \leq 1$ :

$$
D_{t}^{\alpha} X(\mathrm{t})=f_{i}(X(\mathrm{t})), X(0)=X_{0}
$$

where $X(t)=(x(t), y(t), z(t), \varphi(t))^{T} \quad, \quad f(X)=\left[f_{1}(X), f_{2}(X), f_{3}(X), f_{4}(X)\right]^{T}, f_{i}(X)=$ $(x, y, z, \varphi)(i=1,2,3,4), E\left(x_{0}, y_{0}, z_{0}, \varphi_{0}\right)$ is fractional-order system equilibrium point, if all the eigenvalues $\lambda_{i}(i=1,2,3, \ldots, n)$ of the Jacobian matrix satisfy the following condition:

$$
\left|\arg \left(\lambda_{i}\right)\right|>\alpha \pi / 2(i=1,2, \ldots, n)
$$

the equilibrium point is asymptotically stable.

Let the right side of Eq. (10) equal to 0, the equilibrium point of the system can be written as $E\left(x_{0}, 1-5 x_{0}^{2}, 4\left(x_{0}+1.6\right), \varphi_{0}\right)$, where $x_{0}$ and $\varphi_{0}$ are determined by Eq. (13)

$$
\left\{\begin{array}{l}
-x_{0}^{3}-2 x_{0}^{2}+\left(k \varphi_{0}-4\right) x_{0}+I-5.4=0 \\
\operatorname{sgn}\left(\varphi_{0}+2\right)+\operatorname{sgn}\left(\varphi_{0}-2\right)-0.5 \varphi_{0}+0.2 x_{0}=0
\end{array}\right.
$$

It can be seen from Eq. (13) that the equilibrium point of the system is related to the external 
excitation current $I$ and the coupling strength $k$. The Jacobian matrix of system (10) at equilibrium point $E$ is expressed as

$$
J_{E}=\left\{\begin{array}{cccc}
a & 1 & -1 & k x_{0} \\
-10 x_{0} & -1 & 0 & 0 \\
0.0084 & 0 & -0.0021 & 0 \\
0.2 & 0 & 0 & b
\end{array}\right\}
$$

where

$$
a=-3 x_{0}^{2}+6 x_{0}+k \varphi_{0}, \quad b=10^{6}\left(1-\tanh ^{2}\left(10^{3}\left(\varphi_{0}+2\right)\right)\right)+10^{6}\left(1-\tanh ^{2}\left(10^{3}\left(\varphi_{0}-2\right)\right)\right)-0.5
$$

The characteristic equation of system (10) at equilibrium point $E$ is

$$
\lambda^{4}+A \lambda^{3}+B \lambda^{2}+C \lambda+D=0
$$

where

$$
\begin{aligned}
& A=\frac{10021}{10000}-b-a \\
& B=10 x_{0}-\frac{10021 b}{10000}-\frac{10021 a}{10000}+a b-\frac{k x_{0}}{5}+\frac{21}{2000} \\
& C=\frac{21 x_{0}}{1000}-\frac{21 b}{2000}-\frac{21 a}{10000}+\frac{10021 a b}{10000}-10 x_{0} b+\frac{21}{2500}-\frac{10021 k x_{0}}{50000} \\
& D=\frac{21 a b}{10000}-\frac{21 b}{2500}-\frac{21 x_{0} b}{1000}-\frac{21 k x_{0}}{50000}
\end{aligned}
$$

With $k=0.1, I=2.7$, and other parameters remain unchanged, the two expressions in Eq. (13) can be graphically expressed, as displayed in Fig.3. As we can see from Fig.3, two curves govern by Eq. (13) have five intersections, where $\varphi_{0}$ is equal to -2 at the point $E_{4}$ and $\varphi_{0}$ is equal to 2 at the point $E_{5}$. However, when $\varphi_{0}=-2$ or 2, Eq. (13) has no solution, therefore, $E_{4}$ and $E_{5}$ are not equilibrium points. The system, thus, has three equilibrium points $E_{1}, E_{2}$ and $E_{3}$.

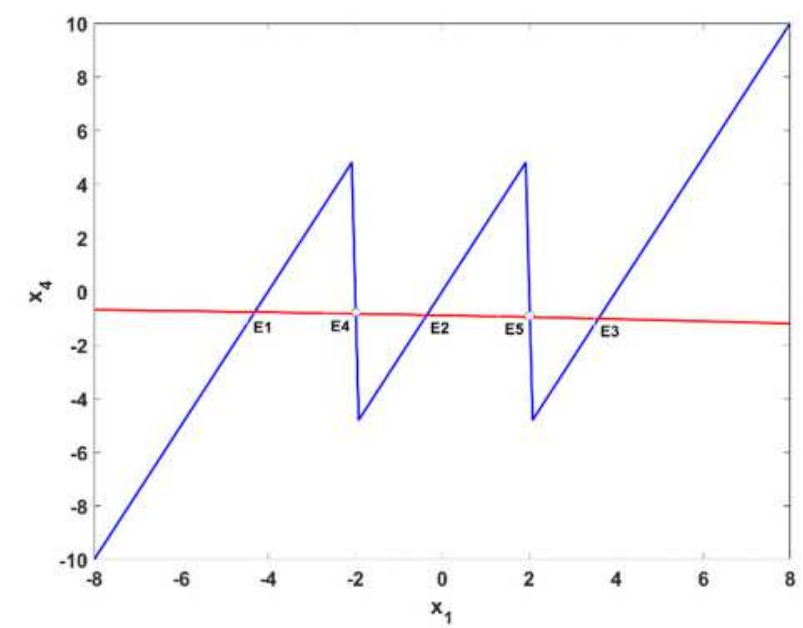

Fig. 3 Two function curves and their intersections, wherein $E_{1}, E_{2}$ and $E_{3}$ are equilibrium points, $E_{4}$ and $E_{5}$ are not equilibrium points.

In order to discuss the influence of external excitation current $I$ and the fractional order $\alpha$ on the stability of the equilibrium points, we list the eigenvalues of the three equilibrium points 
and the relationship between stability and their fractional order under different external excitation current $I=1.27,1.5,1.95$ and 2.7, as shown in Table 1. According to Eq. (15), each equilibrium point has four eigenvalues, and the stability of each equilibrium point is judged by Lemma 1.

According to Lemma 1 and Table 1, we can find when the real parts of the eigenvalues of the equilibrium points are all less than 0 , the neuron is stable and independent of the fractional order $\alpha$. When the eigenvalues of the equilibrium point have both positive real part and imaginary parts, the stability of the equilibrium point is decided by the fractional order $\alpha$. When the eigenvalues of the equilibrium point of the neuron have pure positive real roots, the system is unstable and independent of the fractional order $\alpha$.

Table 1. Equilibrium points and their stability when $k=0.1$

\begin{tabular}{|c|c|c|c|}
\hline$I$ & Equilibrium points & Eigenvalues & Stability analysis \\
\hline 1.27 & $\begin{array}{l}E_{1}(-1.1846,-6.0164,1.6616 \\
\quad-4.4738) \\
E_{2}(-1.3039,-7.5008,1.1844, \\
\quad-0.5215) \\
E_{3}(-1.4440,-9.4257,0.624, \\
\quad 3.4223)\end{array}$ & $\begin{array}{l}\lambda_{1}=-12.7663, \lambda_{2}=-0.4981 \\
\lambda_{3,4}=\mathbf{0 . 0 0 0 0} \pm \mathbf{0 . 0 2 5 6} \boldsymbol{i} \\
\lambda_{1}=-14.0598, \lambda_{2}=-0.4981 \\
\lambda_{3,4}=-0.0018 \pm 0.0245 i \\
\lambda_{1}=-15.5661, \lambda_{2}=-0.4980 \\
\lambda_{3,4}=-0.0076 \pm 0.0226 i\end{array}$ & $\begin{array}{l}\text { The critical Stable } \\
\text { Stable } \\
\text { Stable }\end{array}$ \\
\hline 1.5 & $\begin{array}{l}E_{1}(-1.1268,-5.3483,1.8928, \\
\quad-4.4507) \\
E_{2}(-1.25247,-6.8434,1.3901, \\
\quad-0.5009) \\
E_{3}(-1.3893,-8.6509,0.8427, \\
\quad 3.4442)\end{array}$ & $\begin{array}{l}\lambda_{1}=-12.0333, \lambda_{2}=-0.4981 \\
\lambda_{3,4}=0.0073 \pm 0.0247 i \\
\lambda_{1}=-13.2876, \lambda_{2}=-0.4981 \\
\lambda_{3,4}=0.0063 \pm 0.0237 i \\
\lambda_{1}=-14.7872, \lambda_{2}=-0.4980 \\
\lambda_{3,4}=0.00057 \pm 0.0237 i\end{array}$ & $\begin{array}{c}\alpha<0.817, \text { stable } \\
\alpha>0.817, \text { unstable } \\
\alpha<0.835, \text { stable } \\
\alpha>0.835, \text { unstable } \\
\alpha<0.984, \text { stable } \\
\alpha>0.984, \text { unstable }\end{array}$ \\
\hline 1.95 & $\begin{array}{l}E_{1}(-1.0029,-4.0290,1.2 .3884, \\
\quad-4.4011) \\
E_{2}(-1.1270,-5.3506,1.8920, \\
\quad-0.4508) \\
E_{3}(-1.2674,-70315,1.3304, \\
\quad 3.4931)\end{array}$ & $\begin{array}{l}\lambda_{1}=-10.5250, \lambda_{2}=-0.4982 \\
\lambda_{3,4}=0.0231 \pm 0.0128 i \\
\lambda_{1}=-11.6709, \lambda_{2}=-0.4982 \\
\lambda_{3,4}=\mathbf{0 . 0 2 4 7} \pm \mathbf{0 . 0 0 0 0} i \\
\lambda_{1}=-13.1173, \lambda_{2}=-0.4981 \\
\lambda_{3,4}=0.0197 \pm 0.0129 i\end{array}$ & $\begin{array}{c}\quad \begin{array}{c}<<0.322, \text { stable } \\
\alpha>0.322, \text { unstable }\end{array} \\
\text { The critical unstable }\end{array}$ \\
\hline
\end{tabular}




\begin{tabular}{cll}
\hline$E_{1}(-0.7755,-2.0073,3.2977$, & $\lambda_{1}=-7.9944, \lambda_{2}=-0.4982$ & Unstable \\
$-4.3102)$ & $\lambda_{3}=0.0929, \lambda_{4}=0.00897$ & \\
$\mathbf{2 . 7} \quad E_{2}(-0.5556,-2.9221,2.8572$, & $\lambda_{1}=-8.8307, \lambda_{2}=-0.4983$ & Unstable \\
$-0.3542)$ & $\lambda_{3}=0.1183, \lambda_{4}=0.0058$ & \\
$E_{3}(-1.02219,-4.2244,2.3112$, & $\lambda_{1}=-10.0368, \lambda_{2}=-0.4983$ & Unstable \\
$3.5911)$ & $\lambda_{3}=0.11956, \lambda_{4}=0.00478$ & \\
\hline
\end{tabular}

\section{Dynamic analysis}

In order to study the dynamic behaviors of the neuron, nonlinear dynamical analysis tools such as time series, phase diagram, bifurcation diagram, Lyapunov exponent and ISI bifurcation diagram are used to analyze the firing activities. In the process of numerical analysis, the system parameters are fixed as $a=1, b=3, c=1, d=5, r=0.0021, s=4, \beta=0.2$. It should be noted that Adomian fractional-order algorithm [40] is adopted in this paper, the step length of simulation $h=0.01$, the computing software used is Matlab2019b, and the computer system is based on win10 home edition.

What needs special attention is that many studies have shown that autonomous and nonautonomous fractional-order systems have not any exact periodic solution [41-42]. However, many studies have shown that there exist asymptotically periodic oscillations in fraction-order systems, which are named "Asymptotically T-periodic oscillations" [43-46]. In this paper, we also find the existence of asymptotically periodic oscillations in the proposed fractional-order neuronal model. To facilitate recording, the term "Asymptotically periodic" is written as "Aperiod" in the following.

\subsection{Coexisting firing behavior}

In order to explore the coexisting firing behavior of the fractional-order neuron model, the system parameters are fixed as $k=0.1, \alpha=0.9, I=2.7$ and $\beta=0.2$, the initial values of the fractional-order neuron model are $(1,0,0,-4),(1,0,0,0)$ and $(1,0,0,4)$, the corresponding time series of the membrane potential $x$ are shown in the Figs.4(a)-(c) respectively. From Figs.4(a) and (b), we know $\lim _{t \rightarrow t 1} x_{i}(t+T)-x_{i}(t)=0$ and $\lim _{t \rightarrow t 2} x_{i}(t+T)-x_{i}(t)=0$ are established and the neuron has an A-periodic solution. Although they are both A-periodic solutions, they exhibit different firing patterns. In Fig.4(a) the fractional neuron model exhibits irregular A-periodic bursting and Fig.4(b) illustrates A-periodic-8 bursting. However, as shown in Fig.4(c) when the initial value of the fractional neuron model is $(1,0,0,4), \lim _{t \rightarrow \mathrm{t} 3} x_{i}(t+T)-$ $x_{i}(t) \neq 0$, and thus, the neuron shows a chaotic state. The corresponding phase diagrams are 
illustrated in Fig. 4(d). One can find there exist three different firing patterns with different initial values.

In order to verify the above three different firing patterns, the initial value of the memristor, $\varphi_{0}$, is considered as the controlled parameter and the other parameters remain unchanged, the bifurcation diagram and Lyapunov exponents with respect to the initial value $\varphi_{0}$ are obtained in Fig.5 (a) and (b), respectively. For clarity, the other two Lyapunov exponents LE3 and LE4 in Fig.5 (b) are omitted since their values are much less than 0 . When the initial value $\varphi_{0}$ locates at the region of $(-6,-2)$ and $(-2,2)$, the system is A-periodic, while when the initial value $\varphi_{0}$ is in the region of $(2,6)$, the system presents a chaotic state. From Fig.5 (b), one can find that the maximum Lyapunov exponent is approximately equal to 0 when the initial value $\varphi_{0}$ locates at $(-6,2)$ and $(-2,2)$, meaning the neuron is in a periodic firing state; while the maximum Lyapunov exponent is greater than 0 when the initial value range $\varphi_{0}$ is in $(2,6)$, showing that the neuron produces chaotic firing behavior. So, the bifurcation diagram and the Lyapunov exponents are well consistent.
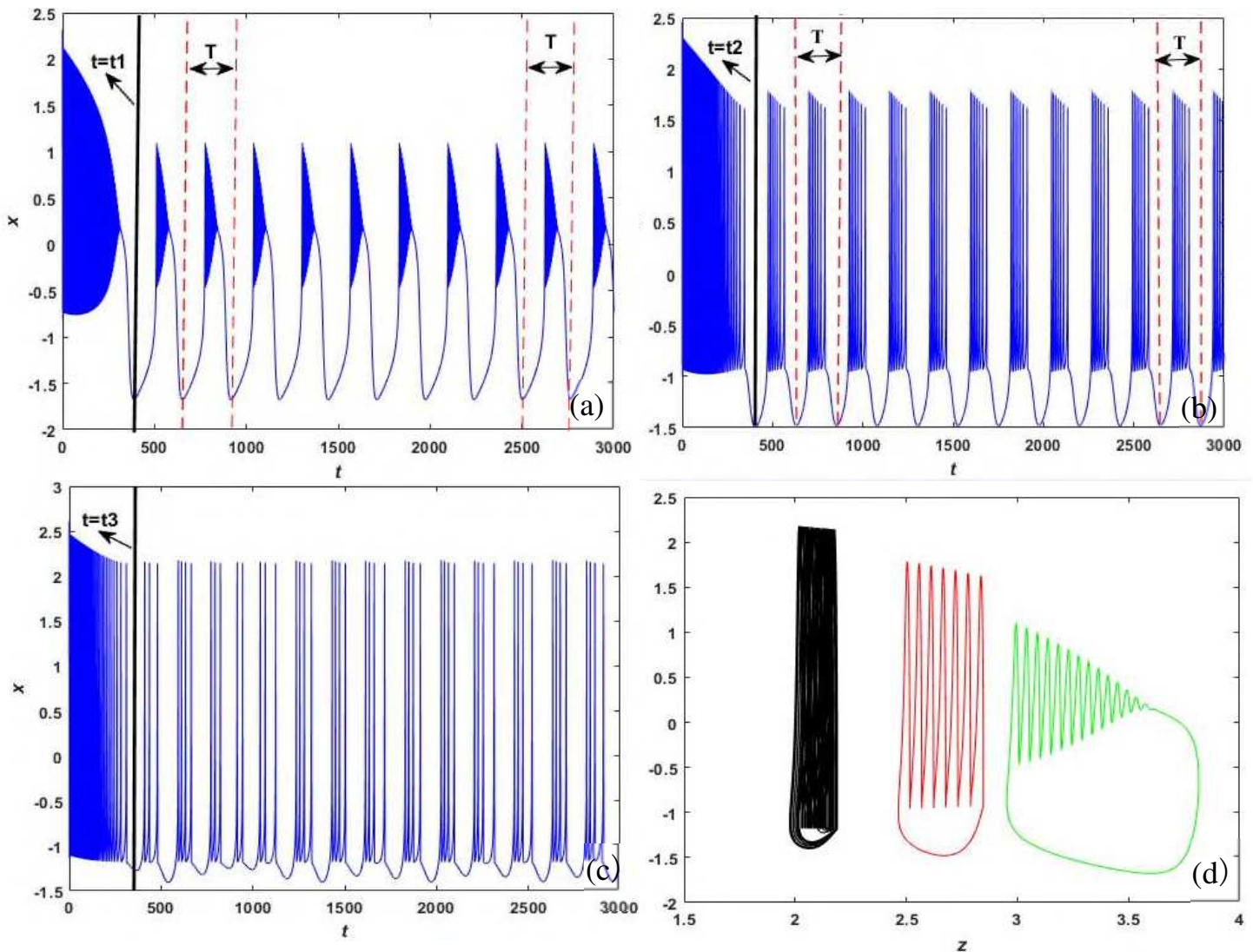

Fig.4 Firing patterns of the fractional-order neuron. (a), (b) and (c) Time series of the membrane potential $x$ with the initial values $(1,0,0,-4),(1,0,0,0)$ and $(1,0,0,4)$, respectively. (d) Phase diagrams, where black, red and green respectively represent the initial value of $(1,0,0,-4),(1,0,0,0)$ and $(1,0,0,4))$. 

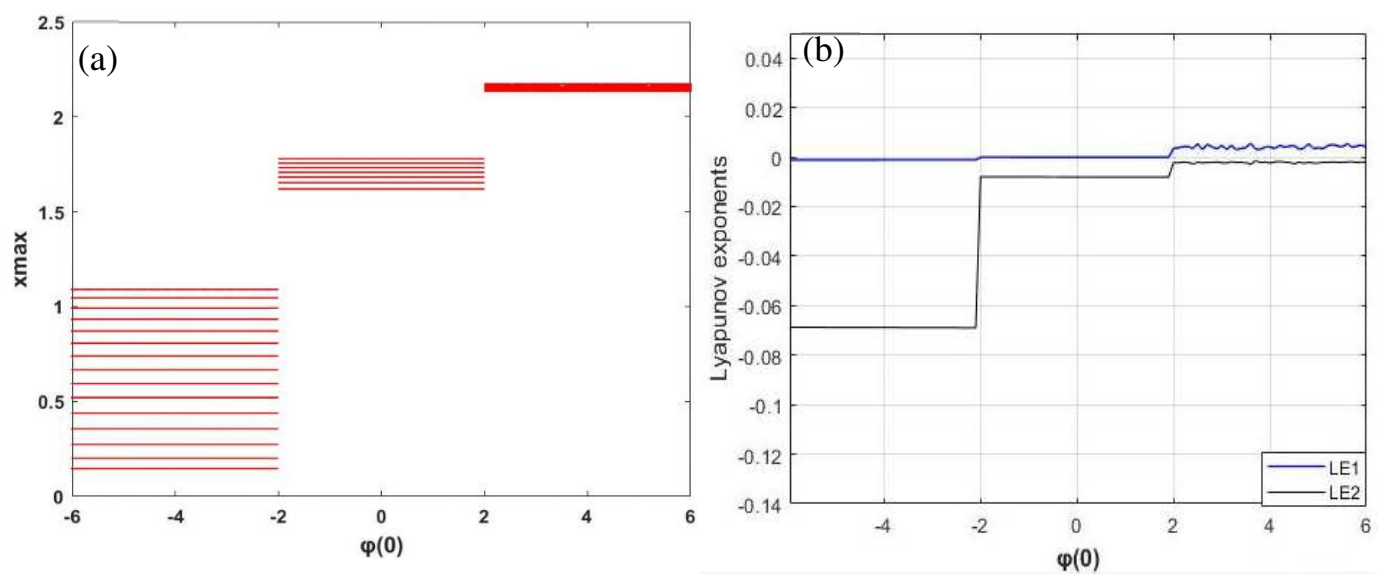

Fig.5 (a) Bifurcation diagram of $x$ with memristor initial value $\varphi_{0}$. (b) Lyapunov exponents with memristor initial value $\varphi_{0}$.

\subsection{Influence of excitation current}

In the real world, neurons respond differently to different external stimuli. In this part, we discuss the effect of the excitation current $I$ on fractional neuron model. Keep $\alpha=0.9, k=0.1$, $\beta=0.2$ and the initial value of the system as $(1,0,0,4)$, when the external excitation current $I$ changes from 1.4 to 3.1, the ISI bifurcation diagram and Lyapunov exponents are shown in Fig.6(a) and (b), respectively.
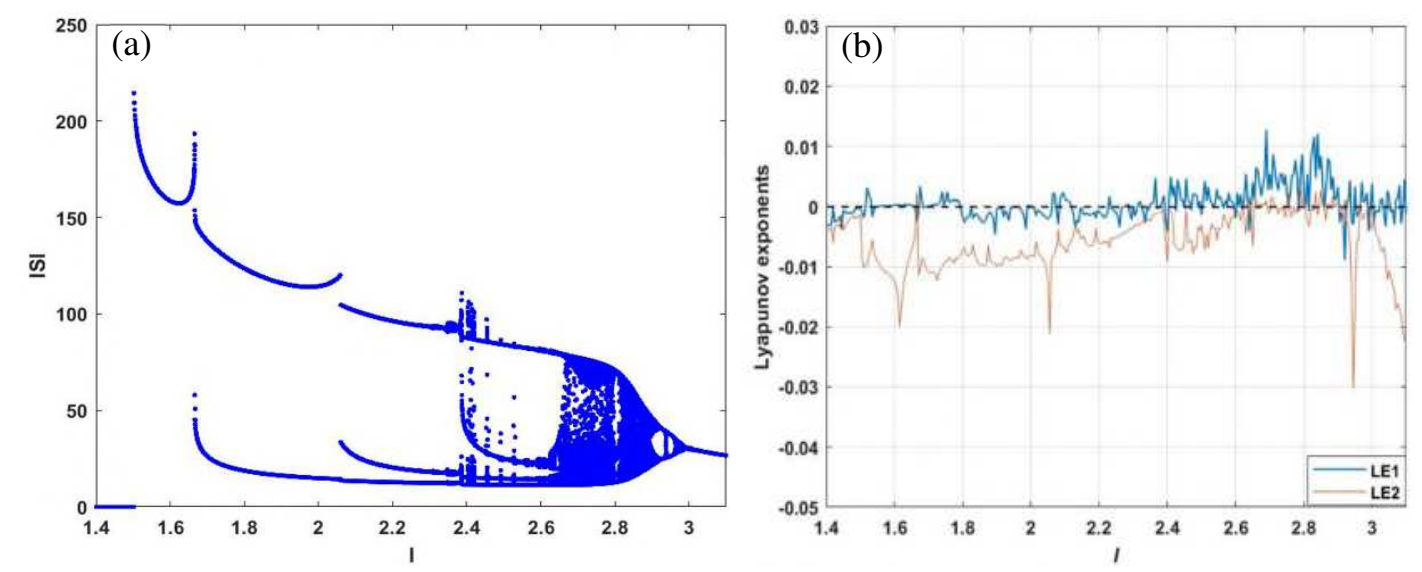

Fig.6 (a) ISI bifurcation diagram and (b) Lyapunov exponents with respect to the external excitation current $I$.

In a fractional-order system, if the trajectory of the system is A-periodic oscillation in some intervals, while the trajectory of other adjacent regions is chaotic, this phenomenon is called "local chaos"[35]. From Fig.6(a), as the external excitation current $I$ increases from 1.5 to 2.6, the fractional-order neuron gradually increases from A-periodic-1 to A-periodic-4. When $I$ increases from 2.6 to 3.1, the neuron appears an A-periodic-chaos-A-periodic switching process. Namely, the neuron presents "local chaos". The Lyapunov exponents of the system are shown 
in Fig.6(b), which are well consistent with the ISI bifurcation diagram.

\subsection{Influence of coupling strength}

Set the fractional order $\alpha=0.9, \beta=0.2, I=2.7$, and the initial value of the system is $(1,0,0,0)$, the coupling strength $k$ is considered as the controlled parameter to explore its influence on the firing dynamics of the neuron model. When the coupling strength $k$ increases from 0.1 to 1.4 , the ISI bifurcation diagram and the corresponding Lyapunov exponents are illustrated in Figs.7 (a) and (b), respectively. From Fig.7(a), when coupling strength $k \in(0,0.5)$, the system is in an A-periodic state, when coupling strength $k \in(0.5,0.95)$, the system alternates between A-periodic and chaotic states, and as the coupling strength $k$ increases from 0.95 to 1.4, the neuron undergoes a series of period doubling bifurcations and finally shows A-period 1 oscillations. Fig.7(b) illustrates the corresponding Lyapunov exponents.
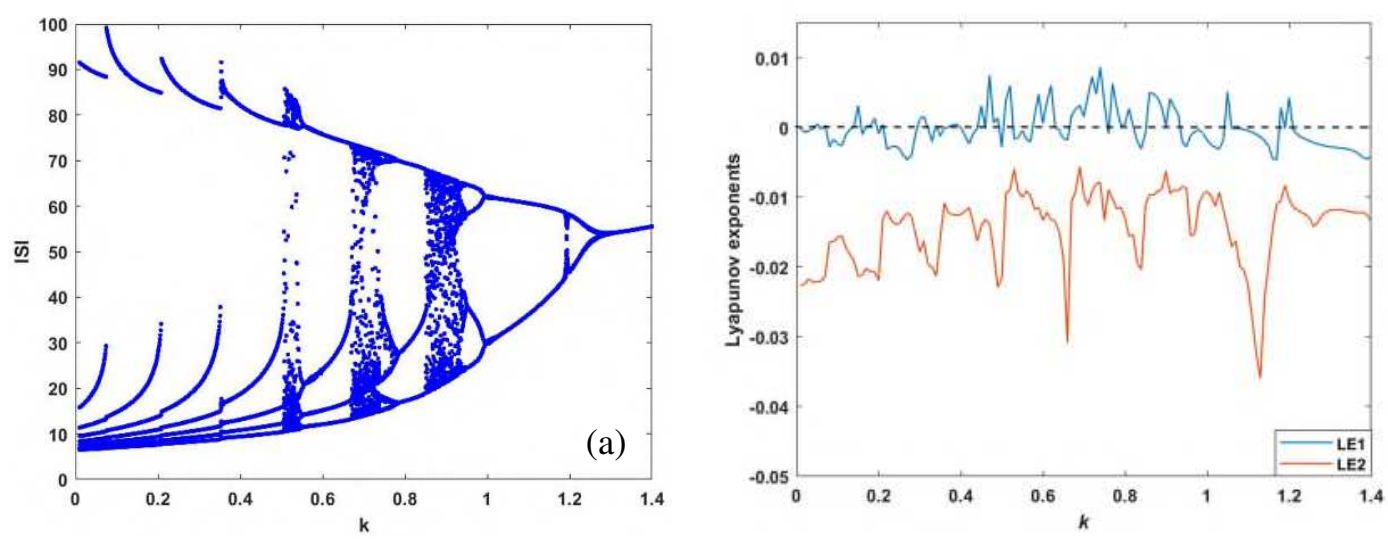

Fig.7 The effect of the coupling strength on firing patterns. (a) ISI bifurcation diagram and (b) Lyapunov exponents. The initial value is $(1,0,0,0)$ and $I=2.7, \alpha=0.9$.

\subsection{Influence of fractional order}

The firing frequency of neuron has great influence on its performance. The more spikes in per unit of time, the faster the action per unit time, and the faster the discharge frequency of neuron. In order to study the effect of fractional order $\alpha$ on the firing frequency of the fractionalorder neuron model, set $k=0.1, \beta=0.2, I=2.7$, and the fractional order $\alpha$ varies from 0.55 to 1 , the ISI bifurcation diagrams of the fractional neuron with the initial value of $(1,0,0,4)$ is show in the Fig.8.

From Fig.8, when $\alpha$ increases from 0.55 to 0.7 , the neuron exhibits A-periodic firing and 
the ISI of the neuron increases gradually, meaning the firing frequency decreases gradually. Figs.9(a) and (b) illustrate the time series of the membrane potential $x$ with the fractional order $\alpha=0.6$ and 0.68 , respectively. In the duration of $t=(1000,1200)$, Fig.9(a) contains 6 complete A-periodic burster, while there are just 4 complete A-periodic burster in Fig.9(b). So, we can intuitively observe that the firing frequency decreases as the increase of the fractional order $\alpha$.

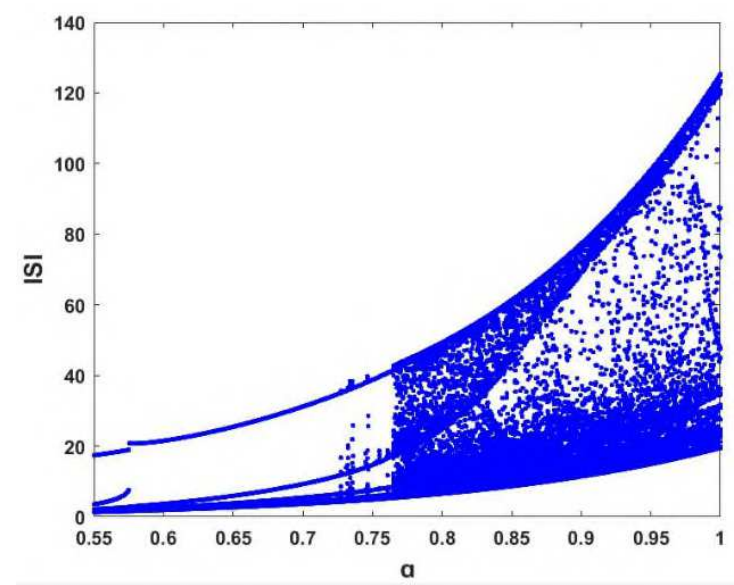

Fig.8 Inter spike interval (ISI) bifurcation diagram with fractional order $\alpha$.

However, from Fig. 8 , as the fractional order $\alpha$ increases from 0.7 to 1 , the firing pattern of the neuron changes from A-periodic to chaotic firing. In order to verify the transition of firing pattern, the time series of the membrane potential $x$ with $\alpha=0.7$ and 0.8 are provided in Figs. 10(a) and (b), respectively. And the corresponding phase diagrams are shown in Fig. 10(c) and (d). It is easy to find when the fractional order is 0.7 , the neuron exhibits A-periodic 5 firing, while the neuron is in chaotic firing state when the fractional order is 0.8 .
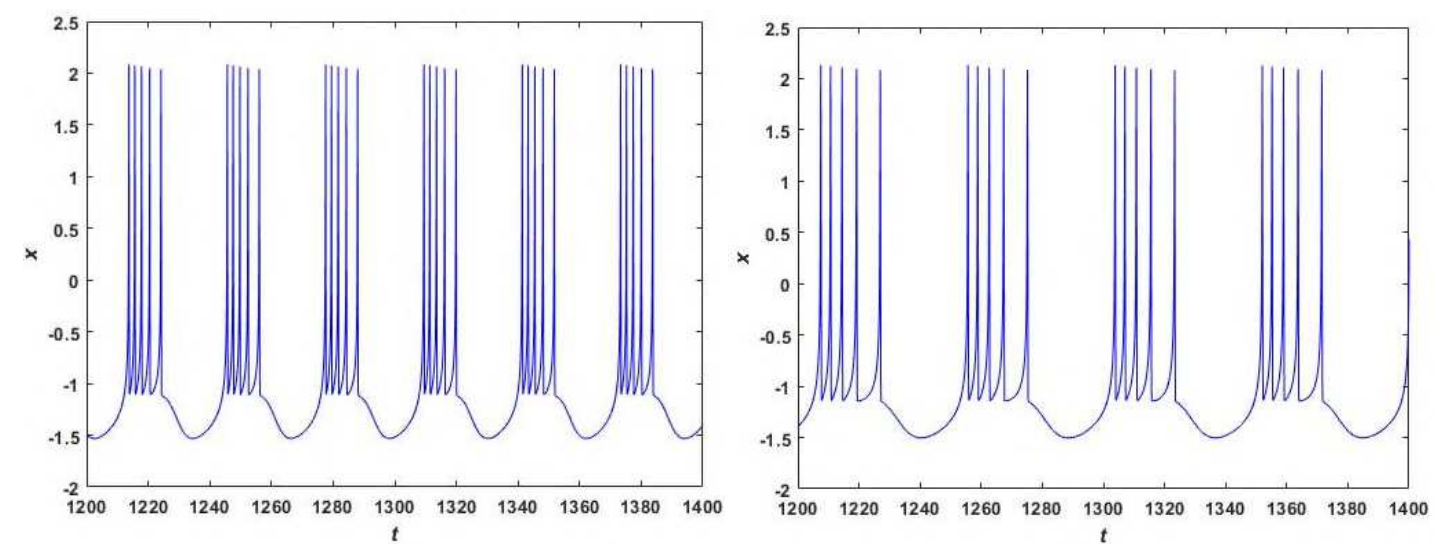

Fig.9 The membrane potential with different orders. (a) $\alpha=0.6$ (b) $\alpha=0.68$. 

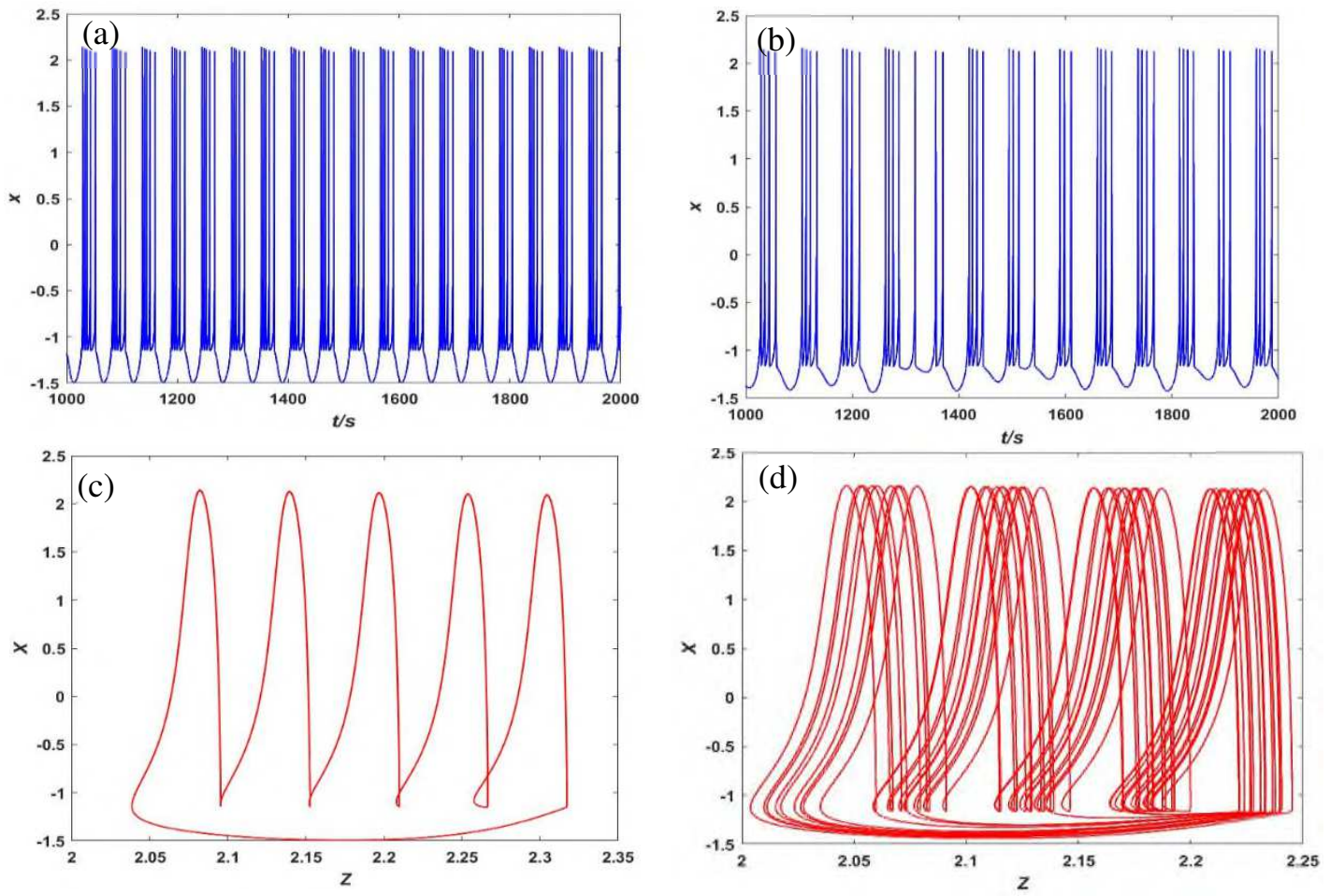

Fig.10 Firing patterns with respect to the fractional order $\alpha$. The initial value of the neuron is $(1,0,0,4), I$ $=2.7$ and $k=0.1$. (a) and (b) represent the time series of the membrane potential with $\alpha=0.7$ and $\alpha=0.8$, respectively, (c)and (d) are the corresponding phase diagrams.

\section{Circuit simulations}

\subsection{Schematics and circuit parameter selections}

In this section, the memristive autapse coupling neuron model controlled by Eq. (10) is realized by using the off-the-shelf electronic components, as shown in Fig.11, in which the operational amplifiers TL082CD and the analog multipliers AD633AN are biased with $\pm 15 \mathrm{~V}$ voltage. From Fig. 11, the complete system circuit consists of three modules: memristor module, $\alpha$ order module and neuron module. It should be noted that $\mathrm{M}$ above the operational amplifier represents the fractional-order adjustable capacitor. According to Kirchhoff's current law, the circuit shown in Fig. 11 can be described by the following equations 


$$
\left\{\begin{array}{l}
\tau_{1} \frac{d^{\alpha} V_{x}}{d t^{\alpha}}=\frac{R_{7}}{R_{8}} V_{y}+\frac{g_{1} R_{7}}{R_{9}} V_{x}^{2}-\frac{g_{1} g_{2} R_{7}}{R_{10}} V_{x}^{3}-\frac{R_{7}}{R_{11}} V_{z}+\frac{R_{7}}{R_{a}} V_{1}+\frac{R_{7}}{R_{b}} V_{x} V_{\varphi} \\
\tau_{2} \frac{d^{\alpha} V_{y}}{d t^{\alpha}}=\frac{R_{12}}{R_{13}} V_{2}-\frac{g_{3} R_{12}}{R_{140}} V_{x}^{2}-\frac{R_{12}}{R_{15}} V_{y} \\
\tau_{3} \frac{d^{\alpha} V_{z}}{d t^{\alpha}}=\frac{R_{16}}{R_{17}} V_{x}+\frac{R_{16}}{R_{18}} V_{3}-\frac{R_{16}}{R_{19}} V_{z} \\
\tau_{3} \frac{d^{\alpha} V_{\varphi}}{d t^{\alpha}}=\frac{R_{1}}{R_{2}}\left(\frac{13.5 R_{f} \operatorname{gggn}\left(V_{\varphi}+2\right)}{R_{3}}+\frac{13.5 R_{f} \operatorname{gggn}\left(V_{\varphi}+2\right)}{R_{4}}-\frac{R_{f} V \varphi}{R_{5}}\right)+\frac{R_{1}}{R_{6}} V_{x}
\end{array}\right.
$$

where four state variables $V_{x}, V_{y}, V_{z}$ and $V_{\varphi}$, denote capacitor voltages of four integral circuit channels, respectively, $\tau_{1}=\tau_{2}=\tau_{3}=\tau_{4}=R_{0} C_{0}$ are time constants of each channel. Comparing the dynamical Eq. (16) with the neuron model (10), when the circuit parameters and system parameters satisfy the following relations

$$
\begin{aligned}
& \frac{R_{7}}{R_{8}}=1, \quad \frac{g_{1} R_{7}}{R_{9}}=a, \quad \frac{g_{1} g_{2} R_{7}}{R_{10}}=b, \quad \frac{R_{7}}{R_{11}}=1, \quad \frac{R_{7}}{R_{a}} V_{1}=I, \quad \frac{R_{7}}{R_{b}}=k, \\
& \frac{R_{12}}{R_{13}} V_{2}=c, \quad \frac{g_{3} R_{12}}{R_{14}}=d, \quad \frac{R_{12}}{R_{15}}=1, \\
& \frac{R_{16}}{R_{17}}=r s, \quad \frac{R_{16}}{R_{18}} V_{3}=1.6 r s, \quad \frac{R_{16}}{R_{19}}=r, \\
& \frac{R_{1}}{R_{2}} \frac{13.5 R_{f}}{R_{3}}=1, \quad \frac{R_{1}}{R_{2}} \frac{13.5 R_{f}}{R_{4}}=1, \quad \frac{R_{f}}{R_{5}}=0.5, \quad \frac{R_{1}}{R_{6}}=\beta,
\end{aligned}
$$

Then the circuit equations (16) are equivalent to those of (10), thus, the proposed circuit can simulate the firing dynamics of the fractional-order neuron. Fixing the system parameters as $a=1, b=3, c=1, d=5, r=0.0021, s=4, \beta=0.2, k=0.1$ and letting $\tau_{1}=\tau_{2}=\tau_{3}=\tau_{4}=100$, one can obtain the following specific values of circuit parameters.

$$
g_{1}=g_{3}=g_{4}=1, R_{0}=10 \mathrm{k} \Omega, C_{0}=1 \mu \mathrm{F}, R_{\mathrm{a}}=R_{1}=R_{7}=R_{8}=R_{9}=R_{11}=R_{12}=R_{13}=R_{15}=R_{16}=
$$

$10 \mathrm{k} \Omega, R_{\mathrm{b}}=100 \mathrm{k} \Omega, R_{6}=50 \mathrm{k} \Omega, R_{f}=1 \mathrm{k} \Omega, R_{3}=R_{4}=13.5 \mathrm{k} \Omega, R_{5}=2 \mathrm{k} \Omega, R_{10}=1 \mathrm{k} \Omega, R_{14}=2 \mathrm{k} \Omega$, $R_{17}=1190.47 \mathrm{k} \Omega, R_{18}=744.047 \mathrm{k} \Omega, \mathrm{R}_{19}=4761.9 \mathrm{k} \Omega, R_{20}=10 \mathrm{k} \Omega, V_{1}=V_{2}=V_{3}=1 \mathrm{~V}$.

According to Ref. [47], the equivalent expression of $\alpha$-order capacitor in complex frequency domain can be written as:

$$
F(\mathrm{~s})=\frac{R_{1}}{s R_{1} C_{1}+1}+\frac{R_{2}}{s R_{2} C_{2}+1}+\ldots+\frac{R_{n}}{s R_{n} C_{n}+1}
$$

where, $n$ is the number of unit integrating units. When fractional-order capacitors with $\alpha=0.7,0.8,0.9$ and 0.98 , according to Refs. [48-49], one can obtain the following 
expressions

$$
F(s)= \begin{cases}\frac{R_{1}}{s R_{1} C_{1}+1}+\frac{R_{2}}{s R_{2} C_{2}+1}+\frac{R_{3}}{s R_{3} C_{3}+1}+\frac{R_{4}}{s R_{4} C_{4}+1}+\frac{R_{5}}{s R_{5} C_{5}+1}+\frac{R_{6}}{s R_{6} C_{6}+1} & \alpha=0.7 \\ \frac{R_{1}}{s R_{1} C_{1}+1}+\frac{R_{2}}{s R_{2} C_{2}+1}+\frac{R_{3}}{s R_{3} C_{3}+1}+\frac{R_{4}}{s R_{4} C_{4}+1}+\frac{R_{5}}{s R_{5} C_{5}+1} & \alpha=0.8 \\ \frac{R_{1}}{s R_{1} C_{1}+1}+\frac{R_{2}}{s R_{2} C_{2}+1}+\frac{R_{3}}{s R_{3} C_{3}+1} & \alpha=0.9 \\ \frac{R_{1}}{s R_{1} C_{1}+1}+\frac{R_{2}}{s R_{2} C_{2}+1} & \alpha=0.98\end{cases}
$$

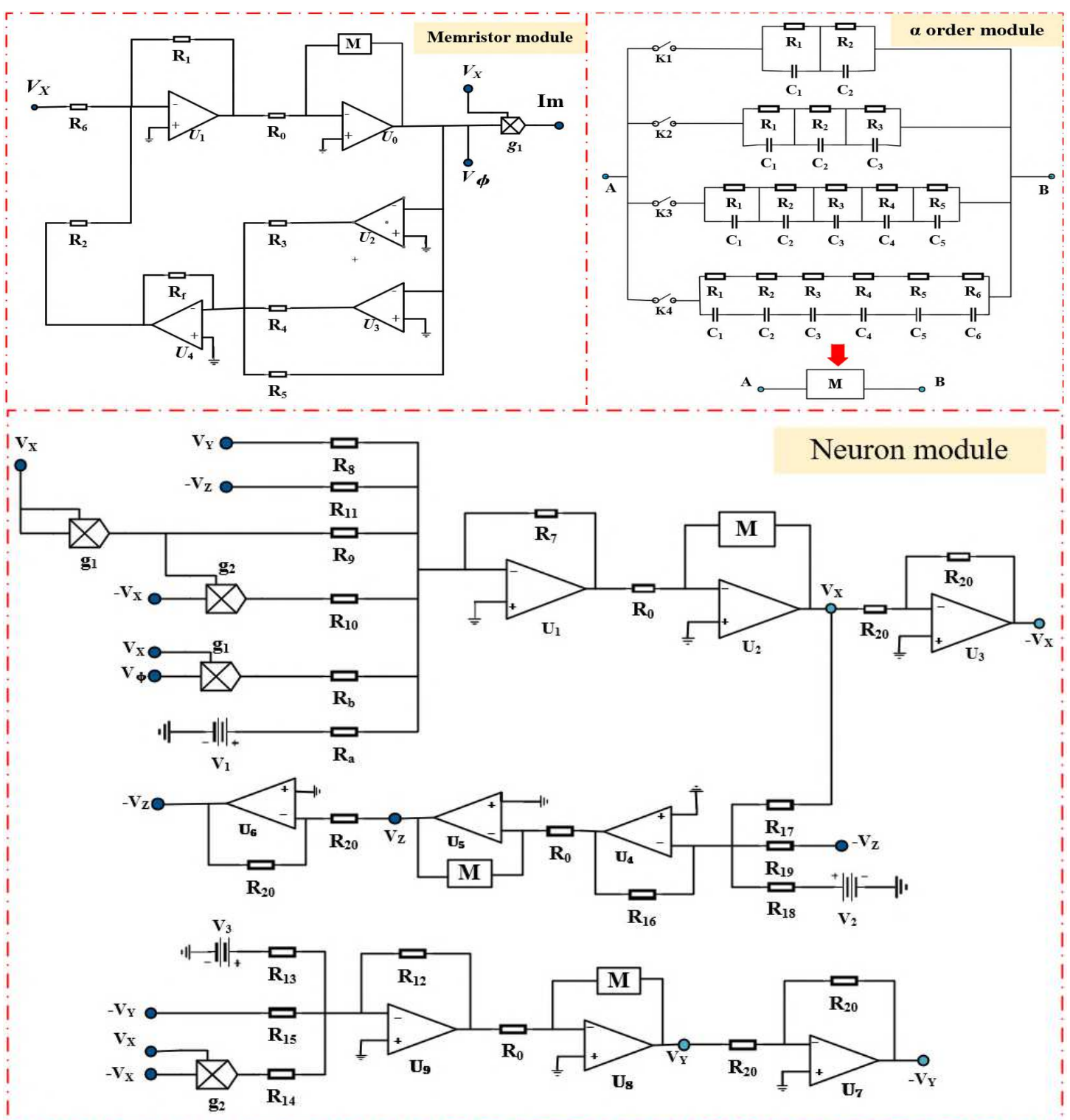

Fig. 11 The main circuit of fractional-order neuron model including memristor module, $\alpha$ order module and neuron module.

The corresponding resistance and capacitance values with different fractional order $\alpha$ are 
listed in Table 2. In the fractional-order capacitor module, the switching elements are used to realize different fractional orders. For example, when the switch $\mathrm{K}_{1}$ is closed and the other switches is turned off, $\mathrm{M}$ is a fractional-order capacitor with $\alpha=0.98$. In this way, we can obtain the other three fractional-order capacitors.

Table 2. The resistance and capacitance values of the $\alpha$-order capacitors.

\begin{tabular}{ccccccccccccc}
\hline $\boldsymbol{\alpha}$ & $\mathbf{R}_{\mathbf{1}} / \mathbf{\Omega}$ & $\mathbf{R}_{\mathbf{2}} / \mathbf{\Omega}$ & $\mathbf{R}_{\mathbf{3}} / \mathbf{\Omega}$ & $\mathbf{R}_{\mathbf{4}} / \mathbf{\Omega}$ & $\mathbf{R}_{\mathbf{5}} / \mathbf{\Omega}$ & $\mathbf{R}_{\mathbf{6}} / \mathbf{\Omega}$ & $\mathbf{C}_{\mathbf{1}} / \boldsymbol{\mu} \mathbf{F}$ & $\mathbf{C}_{\mathbf{2}} / \boldsymbol{\mu} \mathbf{F}$ & $\mathbf{C}_{\mathbf{3}} / \boldsymbol{\mu} \mathbf{F}$ & $\mathbf{C}_{\mathbf{4}} / \boldsymbol{\mu} \mathbf{F}$ & $\mathbf{C}_{\mathbf{5}} / \boldsymbol{\mu} \mathbf{F}$ & $\mathbf{C}_{\mathbf{6}} / \boldsymbol{\mu} \mathbf{F}$ \\
\hline $\mathbf{0 . 7}$ & $2.19 \mathrm{e} 7$ & $2.60 \mathrm{e} 6$ & $5.26 \mathrm{e} 5$ & $1.13 \mathrm{e} 4$ & $2.46 \mathrm{e} 4$ & $6.00 \mathrm{e} 3$ & 3.284 & 3.139 & 1.700 & 0.886 & 0.454 & 0.207 \\
$\mathbf{0 . 8}$ & $3.79 \mathrm{e} 7$ & $1.75 \mathrm{e} 6$ & $1.70 \mathrm{e} 5$ & $1.70 \mathrm{e} 4$ & $1.80 \mathrm{e} 3$ & - & 1.980 & 2.400 & 1.390 & 0.780 & 0.420 & - \\
$\mathbf{0 . 9}$ & $6.284 \mathrm{e} 7$ & $2.50 \mathrm{e} 5$ & $2.50 \mathrm{e} 2$ & - & - & - & 1.232 & 1.835 & 1.1 & - & - & - \\
$\mathbf{0 . 9 8}$ & $9.12 \mathrm{e} 7$ & $1.91 \mathrm{e} 2$ & - & - & - & - & 0.975 & 3.681 & - & - & - & - \\
\hline
\end{tabular}

\section{Circuit simulation results}

Referring to the circuit schematic diagram provided in Fig.11, the fractional-order memristive HR neuron circuit is designed based on Multisim14.0. According to the voltage dividing principle of shunt capacitor, the initial voltage of shunt capacitor is equal to the sum of all shunt capacitors. For the convenience of design, only the initial value of the capacitor $C_{1}$ in the fractional-order capacitor module is changed and the initial values of the other capacitors are set to 0 .

Firstly, we simulate the electrical behavior of fractional-order memristor by using Multisim 14.0. Closing $\mathrm{K}_{2}$ so that the fractional order $\alpha=0.9$, applying a sinusoidal voltage with amplitude $A=2 \mathrm{~V}$ and frequency $F=3.14 \mathrm{~Hz}$ to the fractional-order memristor, we obtain three stable pinched hysteresis loops with different initial values. As illustrated in Fig.12 (a), the red, green and blue hysteresis loops are related to $V_{\mathrm{C} 1}=4 \mathrm{~V}, 0 \mathrm{~V}$ and $-4 \mathrm{~V}$, respectively. Thus, the circuit simulation results effectively verify the numerical simulation results shown in Fig.1(b), indicating that the proposed fractional memristor can indeed exhibit the coexisting multiple stable hysteresis loops. Let $V_{\mathrm{C} 1}=0 \mathrm{~V}$, and close $\mathrm{K}_{4}, \mathrm{~K}_{3}, \mathrm{~K}_{2}$ and $\mathrm{K}_{1}$ sequentially, the corresponding pinched hysteresis loops corresponding to different fractional orders are provided in Fig.11(b), which has the same results as the numerical simulation results as shown in Fig. 1(c). 

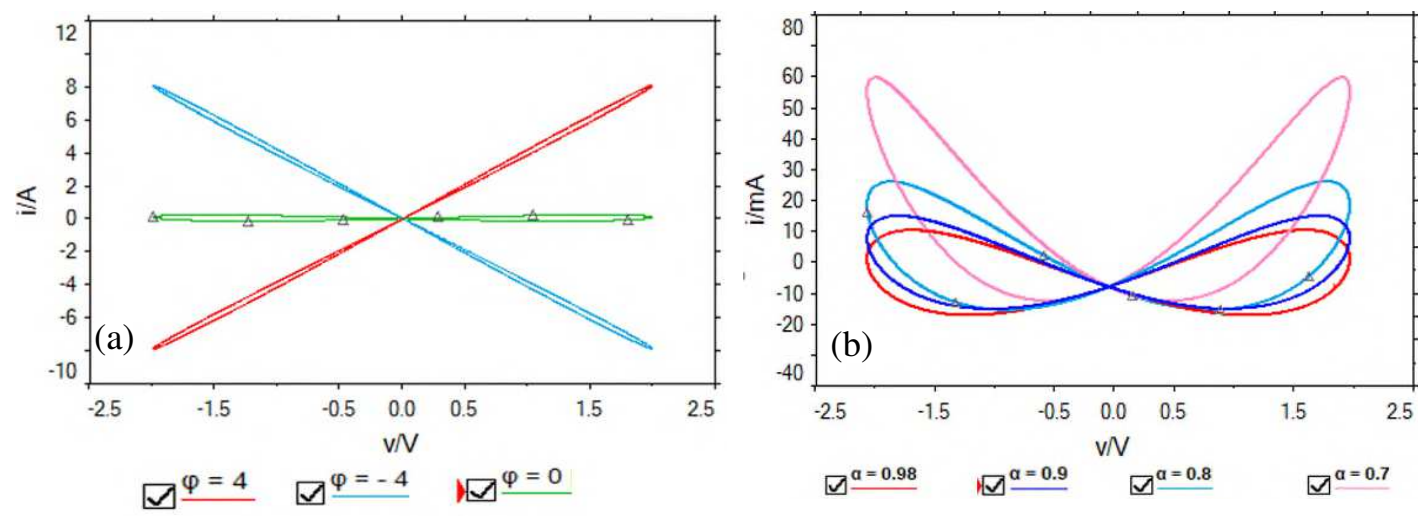

Fig 12. The pinched hysteresis loops obtained by circuit simulation. (a) three stable hysteresis loops with different initial values, (b) Hysteresis loops with different fractional orders.

Secondly, the coexisting firing patterns are also verified by Multisim circuit simulation. close $\mathrm{K}_{2}$ so that the fractional order $\alpha=0.9$, let $V_{\mathrm{C} 1}=-4 \mathrm{~V}, 0 \mathrm{~V}$ and $4 \mathrm{~V}$, respectively, the corresponding time series of the membrane potential $x$ with different initial values are shown in Figs.13(a)-(c) and the corresponding phase portraits in $z-x$ plane are illustrated in Fig.13(d). From Fig.13, one can find that the neuron circuit exhibits three coexisting firing patterns, namely, irregular A-periodic bursting, A-periodic bursting and chaotic firing, which are well consistent with the numerical simulation results illustrated in Fig.4.
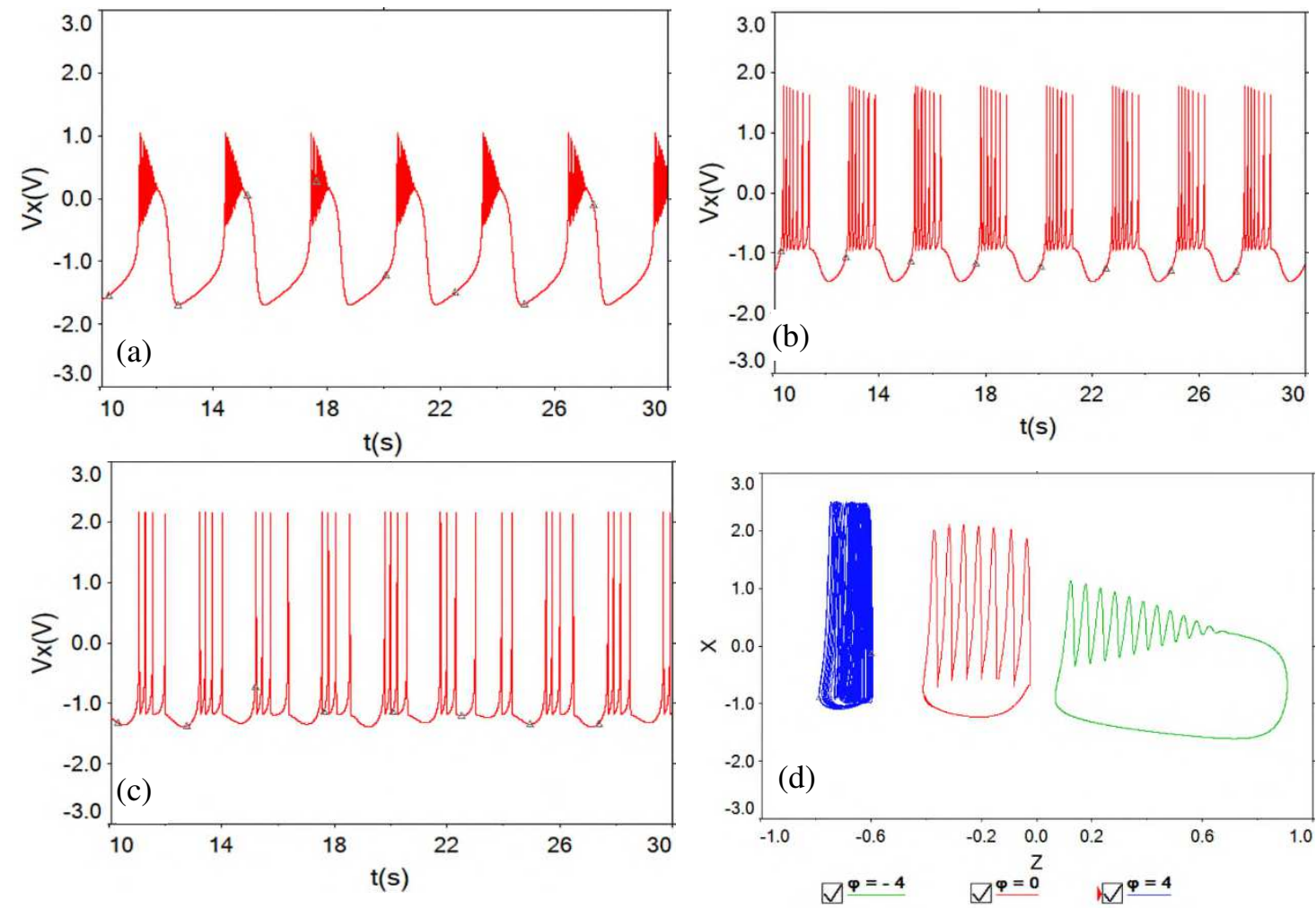

Fig 13 The coexisting firing patterns in circuit simulation. (a)-(c) the time series of the membrane potential with $\mathrm{V}_{\mathrm{C} 1}=-4 \mathrm{~V}, 0 \mathrm{~V}$ and $4 \mathrm{~V}$, respectively, and (d) phase diagram in z-x plane. 
Finally, the firing activities of the neuron circuit dependent on different fractional orders are plotted in Fig.14. For simplicity, we only consider two cases, namely, $\alpha=0.7$ and $\alpha=0.8$. When only the switch $\mathrm{K}_{4}$ is closed, the corresponding fractional order is $\alpha=0.7$, we find the neuron circuit exhibits A-periodic bursting with 5 spikes, the corresponding time series and phase diagram are plotted in Fig.14 (b) and (a). While $\mathrm{K}_{3}$ is on, which means $\alpha=0.8$, we can observe complex chaotic firing activities, as illustrated in Fig.14 (c) and (d). From the numerical simulation results in Fig. 10 and the circuit simulation results in Fig. 14, it is easy to find that the fractional order has an important effect on the firing activities of the neuron.
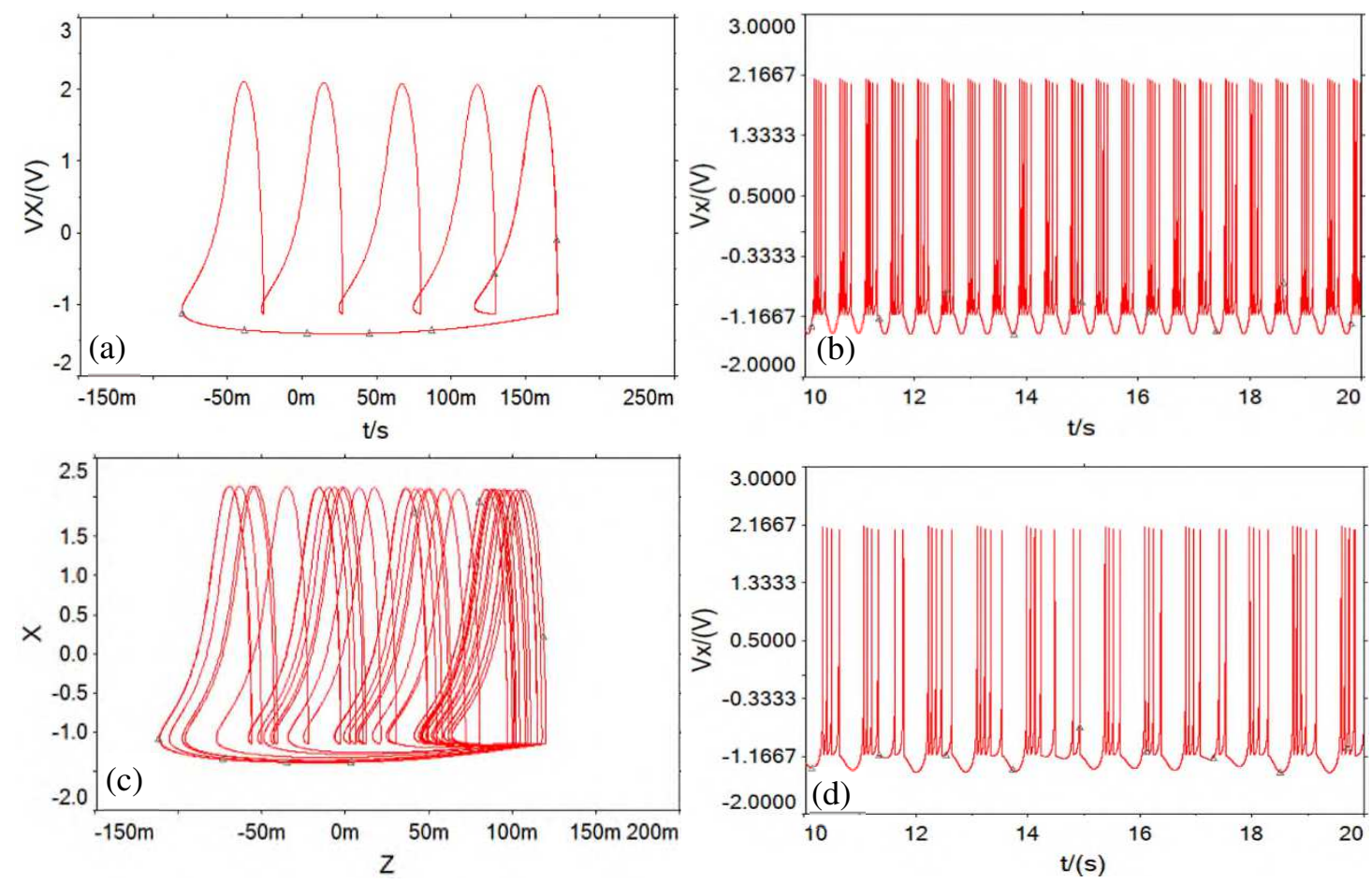

Fig.14 The fractional order dependent firing activities. (a) the time series and (b) phase diagram with $\alpha=0.7$, (c) the time series and (d) phase diagram with $\alpha=0.8$.

\section{Conclusions}

In this paper, a multistable fractional-order locally active memristor is proposed, and its nonvolatile and locally active characteristics are studied and analyzed. Then, a 4D fractional order HR neuron model is developed by introducing the designed memristor as an autapse of a fractional order 3D HR neuron model. The effects of initial condition, coupling strength, external excitation current and fractional-order $\alpha$ on the firing activities of the fractional-order neuron model are discussed in detail by numerical and circuit simulations. Some complex firing 
phenomena including the coexisting firing patterns ignited by different initial values, alternation of A-periodic and chaotic firing induced by coupling strength, are observed. Furthermore, we find that the fractional order $\alpha$ can not only affect the firing patterns but also change the firing frequency of the neuron. It is expected that this study will be helpful to the theoretical analysis and understanding of fractional-order local active memristor and the firing activities of fractional-order neuron.

Acknowledgements This work was supported by the National Key Research and Development Program of China under Grant No.2018AAA0103300 and the National Natural Science Foundations of China under Grant No. 62171401 and 62071411.

\section{Declarations}

Data Availability The datasets generated during and/or analyzed during the current study are available from the corresponding author on reasonable request.

Declaration of Competing Interest The authors declare that they have no known competing financial interests or personal relationships that could have appeared to influence the work reported in this paper.

\section{References}

1. Hodgkin, A.L., Huxley, A.F.: A quantitative description of membrane current and its application to conduction and excitation in nerve. Physiology. 117(4), 500-544 (1952)

2. Hindmarsh, J.L., Rose, R.M.: A model of neuronal bursting using three coupled first order differential equations. Royal society of London. Series B. Biological sciences. 221(1222), 87-102 (1984)

3. FitzHugh, R.: Impulses and physiological states in theoretical models of nerve membrane. Biophysical journal. 1(6), 445-466 (1961)

4. Izhikevich, E.M.: Which model to use for cortical spiking neurons?. IEEE transactions on neural networks. 15(5), 1063-1070 (2004)

5. Morris, C., Lecar, H.: Voltage oscillations in the barnacle giant muscle fiber. Biophysical journal. 35(1), 193$213(1981)$

6. Strukov, D.B., Snider, G.S., Stewart, D.R., Williams, Stanley, R.: The missing memristor found. Nature. 453(7191), 80-83 (2008)

7. Leon, C.: Everything you wish to know about memristors but are afraid to ask. Radioengineering. 24(2), 319 
(2015)

8. Wang, C., Xiong, L., Sun, J., Yao, W.: Memristor-based neural networks with weight simultaneous perturbation training. Nonlinear Dynamics. 95(4), 2893-2906 (2019)

9. Mannan, Z.I., Adhikari, S.P., Yang, C., Budhathoki, RK., Kim, H., Chua, L.: Memristive imitation of synaptic transmission and plasticity. IEEE transactions on neural networks and learning systems. 30(11), 3458-3470 (2019)

10. Chen, C., Bao, H., Chen, M., Xu, Q., Bao, B.: Non-ideal memristor synapse-coupled bi-neuron Hopfield neural network: Numerical simulations and breadboard experiments. AEU-International Journal of Electronics and Communications. 111, 152894 (2019)

11. Bao, H., Zhang, Y., Liu, W., Bao, B.: Memristor synapse-coupled memristive neuron network: synchronization transition and occurrence of chimera. Nonlinear Dynamics. 100(1), 937-950 (2019)

12. Xu, Y., Jia, Y., Ma, J., Alsaedi, A., Ahmad, B.: Synchronization between neurons coupled by memristor. Chaos, Solitons \& Fractals. 104, 435-442 (2017)

13. Ma, J., Wu, F., Wang, C.: Synchronization behaviors of coupled neurons under electromagnetic radiation. International Journal of Modern Physics B. 31(2), 1650251 (2017)

14. Bao, H., Liu, W., Hu, A.: Coexisting multiple firing patterns in two adjacent neurons coupled by memristive electromagnetic induction. Nonlinear Dynamics. 95(1), 43-56 (2019)

15. Tripathi, D., Pandey, SK., Das, S.: Peristaltic flow of viscoelastic fluid with fractional Maxwell model through a channel. Applied Mathematics and Computation. 215(10), 3645-3654 (2010)

16. Hilfer, R.: Applications of fractional calculus in physics. World scientific. (2000)

17. Monje, C.A., Chen, Y.Q., Vinagre, B.M., Xue, D.: Fractional-order systems and controls: fundamentals and applications. Springer Science \& Business Media. (2010)

18. Lundstrom, BN., Higgs, MH., Spain, W.J., Fairhall, A.L.: Fractional differentiation by neocortical pyramidal neurons. Nature neuroscience. 11(11), 1335-1342 (2018)

19. Magin, R.L.: Fractional calculus in bioengineering, part 1. Critical Reviews ${ }^{\mathrm{TM}}$ in Biomedical Engineering, 32(1) (2014)

20. Yu, Y., Shi, M., Kang, H., Chen, M., Bao, B.: Hidden dynamics in a fractional-order memristive HindmarshRose model. Nonlinear Dynamics. 100(1), 891-906 (2020)

21. Alidousti, J., Ghaziani, R.K.: Spiking and bursting of a fractional order of the modified FitzHugh-Nagumo neuron model. Mathematical Models and Computer Simulations. 9(3), 390-403 (2017)

22. Meng, F., Zeng, X., Wang, Z.: Dynamical behavior and synchronization in time-delay fractional-order coupled neurons under electromagnetic radiation. Nonlinear Dynamics. 95(2), 1615-1625 (2019)

23. Meng, F., Zeng, X., Wang, Z.: Adaptive synchronization of fractional-order coupled neurons under electromagnetic radiation. International Journal of Bifurcation and Chaos. 30(03). 2050044 (2020)

24. Malik, S.A., Mir, A.H.: Synchronization of Fractional Order Neurons in Presence of Noise. IEEE/ACM Transactions on Computational Biology and Bioinformatics. (2020)

25. Leng, Y., Yu, D., Hu, Y., Yu, S.S., Ye, Z.: Dynamic behaviors of hyperbolic-type memristor-based Hopfield neural network considering synaptic crosstalk. Chaos: An Interdisciplinary Journal of Nonlinear Science. 30(3), $033108(2020)$

26. Chua, L.O.: Local activity is the origin of complexity. International journal of bifurcation and chaos. 15(11), 3435-3456 (2005)

27. Chua, L.: If it's pinched it's a memristor. Semiconductor Science and Technology.29(10), 104001 (2014)

28. Gibson, G.A., Musunuru, S., Zhang, J., Vandenberghe, K., Lee, J., Cheng, C.: An accurate locally active memristor model for S-type negative differential resistance in $\mathrm{NbO}_{\mathrm{x}}$. Applied Physics Letters. 108(2), 023505 
(2016)

29. Li, Z., Guo, Z., Wang, M., Ma, M.: Firing activities induced by memristive autapse in Fitzhugh-Nagumo neuron with time delay. AEU-International Journal of Electronics and Communications, 153995 (2021)

30. Jin, P., Wang, G., Lu, H., Fernando, T.: A locally active memristor and its application in a chaotic circuit. IEEE Transactions on Circuits and Systems II: Express Briefs. 65(2), 246-250 (2017)

31. Zhu, M., Wang, C., Deng, Q., Hong, Q.: Locally active memristor with three coexisting pinched hysteresis loops and its emulator circuit. International Journal of Bifurcation and Chaos. 30(13), 2050184 (2020)

32. Li, Z., Zhou, H., Wang, M., Ma, M.: Coexisting firing patterns and phase synchronization in locally active memristor coupled neurons with HR and FN models. Nonlinear Dynamics. 104(2), 1455-1473 (2021)

33. Lin, H., Wang, C., Sun, Y., Wei, Y.: Firing multistability in a locally active memristive neuron model. Nonlinear Dynamics.100,3667-3683 (2020)

34. Li, Z., Zhou, H.: Regulation of firing rhythms in a four-stable memristor-based Hindmarsh-Rose neuron. Electronics Letters. (2021)

35. Xie, W., Wang, C., Lin, H.: A fractional-order multistable locally active memristor and its chaotic system with transient transition, state jump. Nonlinear Dynamics. 1-19 (2021)

36. Yang, N., Xu, C., Wu, C., Jia, R., Liu, C.: Fractional-order cubic nonlinear flux-controlled memristor: theoretical analysis, numerical calculation and circuit simulation. Nonlinear Dynamics. 97(1), 33-44 (2019)

37. Leon, C.: Everything you wish to know about memristors but are afraid to ask. Radioengineering. 24(2), 319 (2015)

38. Chang, H., Wang, Z., Li, Y., Chen, G.: Dynamic analysis of a bistable bi-local active memristor and its associated oscillator system. International Journal of Bifurcation and Chaos. 28(08), 1850105 (2018)

39. Matignon, D.: Stability results for fractional differential equations with applications to control processing//Computational engineering in systems applications. 2(1), 963-968 (2016)

40. Adomian, G.: A review of the decomposition method in applied mathematics. Journal of mathematical analysis and applications. 135(2), 501-544 (1988)

41. Tavazoei, MS., Haeri, M.: A proof for non-existence of periodic solutions in time invariant fractional order systems. Automatica. 45(8), 1886-1890 (2009)

42. Kaslik, E., Sivasundaram, S.: Non-existence of periodic solutions in fractional-order dynamical systems and a remarkable difference between integer and fractional-order derivatives of periodic functions. Nonlinear Analysis: Real World Applications. 13(3), 1489-1497 (2012)

43. Danca, M.F., Fečkan, M., Kuznetsov, N.V., Chen, G.: Complex dynamics, hidden attractors and continuous approximation of a fractional-order hyperchaotic PWC system. Nonlinear Dynamics. 91(4), 2523-2540(2018)

44. Danca, M.F, Fečkan, M., Chen, G.: Impulsive stabilization of chaos in fractional-order systems. Nonlinear Dynamics. 89(3), 1889-1903 (2017)

45. Kang, Y.M., Xie, Y., Lu, J.C., Jiang, J.: On the nonexistence of non-constant exact periodic solutions in a class of the Caputo fractional-order dynamical systems. Nonlinear Dynamics. 82(3), 1259-1267 (2015)

46. Danca, M.F., Kuznetsov, N.: Matlab code for Lyapunov exponents of fractional-order systems. International Journal of Bifurcation and Chaos. 28(05), 1850067 (2018)

47. Chen, X., Liu, C., Wang, F.: Circuit realization of the fractional-order unified chaotic system. Chinese Physics B. 17(5), 1664 (2008)

48. Shao, S., Min, F., Ma, M.: Realization and application of fractional Order Chua's System dislocation synchronous non-inductive modular Circuit. Journal of physics. 62(13),130504-1-8. (2013) Chinese

49. Zhou, C., Li, Z., Xie, F.: Coexisting attractors, crisis route to chaos in a novel 4D fractional-order system and variable-order circuit implementation. The European Physical Journal Plus. 134(2), 1-16 (2019) 